\title{
Modelo de Comunicación desde una Perspectiva Social, orientado a la Actividad Física
}

\author{
Adriana Prieto-Rodríguez ${ }^{1}$, Marisol Moreno- Angarita $^{2}$ y Yency S. \\ Cardozo-Vásquez ${ }^{3}$ \\ ${ }^{1}$ Fisioterapeuta. M. Sc. Salud Pública. Departamento del Movimiento Corporal Humano, \\ Facultad de Medicina. Universidad Nacional.E-mail: aprietor@unal.edu.co \\ 2. Fonoaudiologa. Ph. D. (Candidata). M. Sc. Comunicación. Departamento de la \\ Comunicación Humana,. Facultad de Medicina, Universidad Nacional de Colombia. E-mail: \\ mmorenoa@unal.edu.co \\ 3 Fisioterapeuta. Laboratorio del Movimiento Corporal Humano. Facultad de Medicina. \\ Universidad Nacional de Colombia. E-mail: yscardozov@unal.edu.co
}

Recibido 10 Agosto 2005/Enviado para Modificación 14 Diciembre 2005/Aceptado 31 Enero 2006

\section{RESUMEN}

Objetivo Se diseñó y aplicó un modelo de comunicación, en el contexto de una Red aplicada a tres regiones de Colombia, Bogotá, Antioquia y Quindío, basado en el enfoque macrointencional, dirigido a afianzar la comprensión del tema de actividad física en los actores relacionados con el tema desde una interpretación multidimensional.

Métodos Se definieron poblaciones objetivo y se conformaron equipos de trabajo durante un proceso de tres meses, basados en tres estrategias: producción social, transmisión y democratización.

Resultados Se desarrollaron mensajes basados en la construcción de los productores sociales; se ampliaron los conceptos iniciales relacionados con cuerpo, autocuidado, actividad física y salud.

Conclusiones Los modelos de comunicación en salud, que se orientan al desarrollo de habilidades personales en relación con la capacidad de comunicar y construir sentidos compartidos, permiten apropiación y recordación de mensajes en salud. El modelo es una estrategia de comunicación que afianza la construcción compartida de mensajes en salud. Este enfoque exige el desarrollo de acciones locales y el fomento de la capacidad organizacional comunitaria.

Palabras Clave: Comunicación, medios de comunicación, comunicación persuasiva, comunicación social, actividad motora (fuente: DeCS, BIREME).

ABSTRACT

Design of a communicative model from a social perspective oriented to the physical activity case 
REVISTA DE SALUD PUBLICA • Volumen 8 (Sup. 2), Noviembre 2006

Objective A communication model was designed and put into practice, in the form of a Network throughout three regions in Colombia; Bogotá, Antioquia and Quindío. Based on a macro-intentional model, this network was aimed at strengthening understanding around the subject of physical activity among those people affected by the issue, from a multidimensional perspective.

Methods The test population was defined and working groups were formed around three strategies: social production, transmission and democratization, during a three-month period.

Results Messages were developed based around the ideas of the community producers themselves; the initial concepts were widened to include the body, self care, physical activity and health.

Conclusions Communication models related to health, aimed at developing personal skills including the ability to communicate and build shared experience, can be assimilated and incorporated into broadcasts on health issues. This model serves as a communication strategy which strengthens the building of shared broadcasts on health issues. This kind of focus requires the development of local activity and capacity-building within the community.

Key Words: Communication, communications media, persuasive communication (source: $\mathrm{MeSH}, \mathrm{NLM}$ ).

L a comunicación es una estrategia ampliamente identificada en las acciones de cuidado y protección en salud. Un primer enfoque se relaciona con el dominio de información y su importancia, oportunidad, credibilidad, validez y por la adecuación de la propuesta a las percepciones, expectativas y lenguajes de aquellos con quienes se busca convencer. Un segundo enfoque la orienta como proceso social a escala multitudinaria, dirigida al desarrollo de conocimientos que forjan actitudes y provocan prácticas de salud favorables (1).

La transmisión masiva de información ha sido uno de los enfoques en salud más tradicionales (2), en parte por la comprensión de los comportamientos y la explicación de su transformación y por el desarrollo tecnológico de estos medios. Sin embargo, se observa con mayor claridad cada vez, que el proceso de comunicación en salud, implica la construcción de identidades y que por tanto la información debe ajustarse a la realidad de lenguajes y contextos locales en que los sujetos se desenvuelven (3-4).

En general para América Latina la comunicación en salud se ha enmarcado bajo tres diferentes tendencias (5): En primer lugar, como instrumento, herramienta, medios, en donde el énfasis se centra en la comunicación masiva, donde comunicar es transmitir información; en segundo lugar, como eje 
de los procesos de organización, participación y cambio social, en ella el énfasis se presenta en la comunicación política: grupal, popular, liberadora; y en tercer lugar, una concepción híbrida, entendida como red, como tejido que se constituye en fundamento de la cultura e interacción humana.

En la primera tendencia, se desarrollan modelos de comunicación y experiencias como el mercadeo social (6) como cambio motivador en el comportamiento y el mercadeo de productos y servicios necesarios para la salud, en el cual existen experiencias en actividad física, como la campaña VERB (7), en la cual se han aplicado para el fomento de la actividad física, estrategias de producto, precio, lugar y promoción del mensaje.

En la segunda tendencia, orientada al cambio social, existen experiencias como la estrategia COMBI (8-9), en la cual se establece una agenda de comunicación desde un enfoque de movilización social, con elementos como el de participación comunitaria y abogacía. Estos enfoques se orientan a la aplicación de estrategias de comunicación local y popular.

En la tercera tendencia se encuentran ejemplos como el modelo macrointencional (10), el cual se dirige a generar modificaciones en los campos de actuación de los re-editores en función de un propósito colectivo. En esta un productor social, convoca a re-editores sociales determinados, a través de redes de comunicación directa, con apoyo en medios masivos.

El modelo de comunicación que se presenta en este artículo, se inscribió en esta última tendencia y constituyó tres estrategias básicas, en las que se articularon elementos propios del modelo macrointencional, ajustados a una red de movilización social, denominada vida y comunidad en movimiento y desarrollada en tres regiones de Colombia, Bogotá, Antioquia y Quindío. Las acciones de comunicación se dirigieron a favorecer: a. la comprensión del tema de actividad física en los actores relacionados con el tema desde una interpretación multidimensional; b. procesos de información-comunicación local, basada en la propia construcción de los grupos comunitarios, sobre el tema de la actividad física y su relación con el ocio y el trabajo; y, el desarrollo de la estrategia de comunicación desde el enfoque colaborativo y de re-edición, a partir de la integración de actores.

\section{MÉTODOS}

Con base en un análisis de contexto en el que se identificaron las principales necesidades de comunicación en el tema se definieron la población objetivo y los mensajes clave. 


\section{Población objetivo}

Adultos entre 25 y 50 años pertenecientes al régimen contributivo del Sistema General de Seguridad Social.

Comunidades: se tomaron como las colectividades que comparten un espacio territorial (barrio) y algunos intereses por su desarrollo.

Organizaciones que ejercen autoridad local: se refirió a las autoridades de gobierno regional y municipal. En esta categoría también se incluyeron representantes de instituciones del Estado, por ejemplo: Institutos de Recreación y Deporte, Institutos de Cultura y Turismo, Secretarías de Educación, Planeación Distrital y Desarrollo Urbano, Secretarías y seccionales de Salud. Alcaldes, Concejales y ediles. Algunas de estas ya habían efectuado sistemáticamente acciones dirigidas al fomento de la actividad física; otras, lo habían logrado indirectamente; y un pequeño grupo aún no había identificado la pertinencia del tema en sus agendas.

Mensajes clave

- La condición de persona se fundamenta en que se es y se tiene un cuerpo.

- El movimiento corporal y la actividad física, son condiciones propias de la vida, y por tanto deben vivirse.

- La actividad física es un factor protector de la salud, de bajo costo y alta accesibilidad.

- El sedentarismo como un problema social: es responsabilidad de todos.

- Participación de la comunidad.

Estrategias de medios

Se establecieron tres tipos de medios para los desarrollos del mensaje, consistentes en medios masivos, locales y directos.

Medios masivos: los mensajes difundidos a través de este tipo de medios, tuvieron como fin invitar a la audiencia a participar, crear expectativa sobre el tema y motivar a la acción. La población objeto es indiferenciada y el auditorio es universal. Especialmente se usaron canales de prensa y radio. 
Medios locales: se emplearon para el desarrollo de los mensajes en las comunidades a intervenir (grupo estudio), en el contexto de la Red Vida y Comunidad en Movimiento y su principal población objetivo son las comunidades. Se enfatizó en el enfoque de re-editores y desarrollo del modelo macrointencional de generación de productores sociales de la comunicación.

Medios directos: se emplearon para favorecer los procesos de movilización y organización de las poblaciones en relación con el tema, se hizo énfasis en el aspecto comunitario y desde allí se dirigieron iniciativas hacia las autoridades locales y los miembros de la comunidad. Se vinculó con la estrategia de Red Vida y Comunidad en Movimiento y actores sociales para la re-edición.

Áreas y fases

Se definieron tres áreas de desarrollo en el modelo, de producción, transmisión y de democratización. A través de cuatro fases, sensibilización, valoración, apropiación y sostenimiento.

En el área de producción se desarrollaron las formas y objetos de comunicación. Se construyeron por parte de un Productor Social, retomando el conjunto de actores que se encontrarían potencialmente en capacidad de tomar la estrategia de comunicación y desarrollar un proceso sostenible a partir de las piezas de comunicación editadas en el proyecto. En las fases de sensibilización y valoración se desarrollarían las acciones propias del proyecto y las alianzas que se adelantarían. En la fase de apropiación se esperaría integrar en calidad de productores sociales, actores y organizaciones que se permitan la sostenibilidad de la estrategia. Sobre este confluyen el mensaje, el campo de actuación del re-editor, la población de re-editores y la población de incidencia de los re-editores.

El área de transmisión consistió en el proceso de edición de piezas de comunicación, uso de medios, desarrollo del mensaje en cada estrategia mediática y para cada audiencia. Se desarrollaron diferentes contenidos del mensaje y adaptaciones del mismo de acuerdo al perfil de audiencia, la fase y el canal de transmisión.

El área de democratización consistió en un trabajo colaborativo de re-edición. Se efectuaron acciones dirigidas a favorecer la participación de los reeditores, en busca de utilizar la comunicación para facilitar los esfuerzos de las personas, grupos e instituciones interesadas en transformar las condiciones que caracterizan el problema de la actividad física. 
En la fase de sensibilización, se trabajó en involucrar a los actores, favorecer la comprensión del problema de la actividad física y el sedentarismo en un discurso común, apoyar la canalización de información y acceso a la misma y analizar procesos fortalezas y debilidades para el desarrollo de la estrategia de comunicación, así como facilitar el reconocimiento de posibles contribuciones de la estrategia de comunicación.

En la fase de valoración se trabajó con los participantes en la definición de acciones y priorización de las mismas, desarrollar acciones, materiales y recursos de comunicación incluyendo a los actores (re-editores) comunitarios, discutir los productos de comunicación, enriquecer sus discursos y enfoques, aplicándolos al contexto específico e involucrar instancias, recursos y aliados que apoyen el proceso de re-edición y que originalmente no se han interesado.

En la fase de apropiación, se desarrolló la estrategia de comunicación con enfoque colaborativo y de re-edición, definiendo un plan de trabajo y responsabilidades en el equipo de reeditores y de proceso de re-edición y el empleo y ajuste de tácticas de comunicación.

Finalmente en la fase de sostenimiento, se trabajó en intercambiar experiencias para mejorar acciones futuras y mejorar la capacidad de toma de decisiones en los grupos.

\section{RESULTADOS}

En relación con la producción, transmisión y democratización del mensaje, se desarrollaron diversas acciones a través de medios directos, locales y masivos. A continuación se exponen las actividades y posteriormente la valoración que las comunidades participantes hicieron del proceso a partir de una metodología de sistematización de experiencias.

\section{Actividades}

La comunicación directa fue el principal elemento de trabajo en la intervención a escala individual, en total se desarrollaron 8336 mensajes directos, enviados por medio de correspondencia a las personas encuestadas que conformaron el grupo de estudio en cada región. La herramienta empleada consistió en un boletín informativo el cual contenía cinco secciones relacionadas con actividad física: artículo de la semana, consejos prácticos, pasatiempo, agenda y contáctenos. La recepción de estos mensajes fue de un $85 \%$ aproximadamente. El involucramiento de personas a las demás actividades 
organizadas, como resultado de estos mensajes fue de un $10 \%$ aproximadamente.

Otra estrategia comunicativa, desarrollada en comunicación directa a escala individual, fue la elaboración de una cartilla trabajada por líderes comunitarios, atendiendo a un proceso de democratización de la comunicación. Este trabajo se efectuó en Quindío, abarcando 372 personas.

En los niveles comunitario e institucional, se emplearon como modalidades de comunicación directa las llamadas telefónicas, visitas a actores y líderes específicos, asambleas, reuniones y la ejecución de festivales de movimiento. En cada caso se observó acogida a estas actividades y especialmente se garantizó el involucramiento en la fase de sensibilización a través de ellas.

En la comunicación local, se adelantaron cartillas orientadas a todos los niveles. Entre ellas es necesario resaltar aquellas que fueron trabajadas por los participantes en la Red, especialmente en el Quindío, en donde los procesos de democratización de la comunicación y de re-edición planteados por el modelo, fueron aplicados de manera más precisa. Estos ejercicios de desarrollo de cartillas como productos locales de comunicación contribuyeron, en general, a propiciar un ambiente de confianza en las capacidades de cambio de los grupos al igual que mayor comprensión de los conceptos y construcción colectiva de los mismos. De manera similar desarrollaron un sentido de equidad y oportunidad en la información para procesos de gestión comunitaria. De otra parte, también en el ámbito empresarial se efectuaron mensajes por Intranet orientados al fomento de la actividad física, elaborados por la misma comunidad involucrada en la intervención.

Otros elementos incluidos dentro de la estrategia de comunicación masiva fueron dos videos dirigidos a afianzar conceptos y metodologías para el desarrollo de actividad física, en los grupos conformados. De la misma manera se diseñó un hipertexto dirigido a los miembros de la Red, en el cual se describieron los desarrollos del modelo y sus contenidos.

La modalidad de comunicación por medios masivos se dirigió a un auditorio amplio, conformado por las audiencias que cada medio pautado ofrecía. Específicamente, se efectuaron 119 avisos en prensa, sumando los de cobertura nacional y regional. Se realizaron 323 pautas en televisión y 6154 por radio.

Sistematización de la experiencia 
Se exploró en la comunidad participante la identificación de los aspectos relacionados con su historia local, la construcción de discursos compartidos y la construcción colectiva de conocimientos, en el marco de un proceso de organización comunitaria en Red.

Historia local: los participantes de las comunidades establecieron relaciones entre el proceso vivido de comunicación y sus propias experiencias, ya que los conceptos de comunicación, lenguaje, interacción social y construcción participativa de conocimientos fueron escuchados y asimilados a partir de la experimentación.

Los participantes adoptaron el rol de autores-lectores para evaluar los mensajes de comunicación diseñados para la comunidad teniendo como precedente las características de la audiencia a la cual se dirigieron. Cada comunidad debió establecer sí estos se acoplaban a sus necesidades específicas locales.

También se propició la construcción colectiva de conocimientos para la pieza escrita de comunicación local diseñada por y para las comunidades, acorde con la población objetivo y el propósito de este producto mediático.

Adicionalmente reconocieron en la participación comunitaria y la construcción de conocimientos, formas de organización para dar soluciones a problemas sociales, proporcionando estrategias a ejecutar para lograr los objetivos propuestos.

Dieron importancia a la comunicación como una herramienta que permite el desarrollo social, ya que facilita el reconocimiento y solución de las problemáticas de interés comunitario. Reflexionaron acerca de la importancia que tiene ésta y el uso adecuado del lenguaje para informar e interactuar efectivamente con los demás miembros de la comunidad.

De igual forma, en esta categoría se indagó por la construcción compartida de discursos en la cual se identificó que los participantes proporcionaron ejemplos para sustentar la importancia de la comunicación y agregaron que la deficiencia en ésta, se constituía en uno de los más graves problemas que afrontan sus comunidades para el desarrollo de proyectos, y en consecuencia, para la misma interacción social entre las mismas.

Las personas encontraron similitudes en las representaciones acerca del concepto de actividad física obtenidas y la importancia del uso del lenguaje 
para que los mensajes fueran comprensibles para los demás miembros de su comunidad.

Reconocieron el proceso escritural, como un proceso complejo, difícil de realizar cuando se toman opiniones de varias personas y en ocasiones divergentes; asimismo, reconocieron la importancia de transmitir eficientemente los mensajes escritos para darlos a conocer a los miembros de sus comunidades como una herramienta fundamental para sensibilizar y generar cambios en los estilos de vida llevados por la mayoría de los habitantes de sus comunidades.

Las acciones desarrolladas permitieron realizar una transferencia de los conocimientos construidos participativamente y las herramientas proporcionadas, a otros ámbitos de la vida social, tales como la organización de proyectos personales y comunitarios que buscan resolver las problemáticas diversas que enfrentan las comunidades.

Las herramientas proporcionadas permitieron optimizar los procesos comunicativos en el ámbito comunitario, generar respeto por las opiniones dadas por los miembros de un grupo de trabajo; además, facilitar la interacción social entre miembros de la misma comunidad y de comunidades aledañas.

Construcción de conocimientos: Los participantes desarrollaron conocimientos colectivos en cuanto a estrategias y representaciones para su comunidad del tema. Las estrategias que emergen del discurso colectivo son las que implican la cooperación comunitaria para el desarrollo exitoso de objetivos propuestos para dar solución de las problemáticas que aquejan a las comunidades.

En este sentido, reconocieron que la comunicación verbal y el lenguaje verbal no son las únicas formas de expresión de: ideas, sentimientos y opiniones y que en su cuerpo es una herramienta de comunicación.

Identificaron además que la comunicación permite abarcar gran parte de la comunidad, ya sea de manera verbal o escrita facilita la cobertura de información a las comunidades así como su persuasión, además facilita la interacción social de los miembros de las comunidades y la solución de conflictos.

Por otra parte, establecieron la importancia que la comunicación tiene en el ámbito personal, familiar y comunitario. Realizando reflexiones acerca del uso adecuado del lenguaje para que la intención del mensaje pueda ser 
transmitida, teniendo en cuenta los miembros de la comunidad a los cuales sería transmitido.

Reconocieron la trascendencia de la comunicación para la interacción social y el establecimiento de relaciones interpersonales, fundamentales para el desarrollo en todas las dimensiones en las que se desenvuelve el ser humano y las comunidades; la importancia de la comunicación para el desarrollo comunitario y para la solución de problemas que lo aquejan, reconocimiento de la trascendencia de la comunicación efectiva, es decir, un adecuado uso del lenguaje para comunicar dentro de la comunidad; y la importancia de la construcción participativa de conocimientos y de la comunicación como una estrategia para sensibilizar a las personas de su comunidad acerca de la importancia de la realización de actividad física regular para su salud. Además, la importancia de la construcción participativa de conocimientos para el enriquecimiento de discursos y asimilación de conceptos que benefician a la comunidad y el contexto inmediato de los individuos.

\section{DISCUSIÓN}

Los modelos de comunicación en salud, cada día se orientan más a un enfoque de organización social y conformación de redes $(11,12)$, en los que se articula la participación institucional y comunitaria en un objetivo común. Sin embargo, se encuentran fisuras en el ámbito colombiano relacionadas con este proceso, en primer lugar la predominancia de un enfoque informativo y de masificación (5), y en segundo lugar la competencia en este tipo de modelos de los profesionales de la comunicación (13) situación que en este modelo fue identificada, pues en el desarrollo del plan de trabajo fue necesario un despliegue de medios masivos, aún cuando no fuera clara la efectividad de este y se encontró limitación en el equipo de comunicadores en cuanto a su formación y dominio de programas de comunicación en salud.

Por otra parte, se encuentra que este tipo de programas afianzan procesos de movilización social y se dirigen al desarrollo de acciones de mayor sostenibilidad en la medida en que involucran el desarrollo de la capacidad comunitaria y la abogacía (14). En el presente proyecto este aspecto se identificó en que las acciones desarrolladas permitieron realizar una transferencia de los conocimientos construidos participativamente y las herramientas proporcionadas, a otras actividades de su vida como la organización de proyectos personales y comunitarios para las problemáticas diversas que enfrentan sus comunidades. 
Las herramientas proporcionadas permitieron optimizar los procesos comunicativos en el ámbito comunitario, generar respeto por las opiniones dadas por los miembros de un grupo de trabajo; además, facilitar la interacción social entre miembros de la misma comunidad y de comunidades aledañas.

Este proyecto constituye una experiencia dirigida a la aplicación de estrategias de comunicación orientadas a la promoción de la actividad física desde un enfoque de organización social en Red. En el cual se desarrollaron acciones empleando y articulando medios masivos, locales y directos en un ambiente de democratización.

Se resaltan como principales rasgos del proceso metodológico, los aplicados para el desarrollo de la democratización, En primer lugar, las actividades de tipo grupal en las cuales se propició la construcción participativa de conocimientos; en segundo lugar, la re-edición entre los grupos de trabajo para que se brindaran aportes a la construcción, que sería plasmada en una pieza de comunicación local escrita, la socialización de la construcción grupal y de la re-edición para que si lo consideraban necesario los demás participantes realizarán más aportes y la reunión de opiniones para llegar a un consenso sobre la información que se brindó en la pieza de comunicación escrita diseñada por las comunidades teniendo en cuenta las particularidades de la audiencia objetivo y el objetivo propuesto; en tercer lugar, la construcción crítica de los mensajes de comunicación diseñados para la comunidad para valorar su grado de comprensión, atracción, involucramiento y credibilidad, mediante la aplicación de un formato para la evaluación de mensajes de comunicación en salud en campañas de comunicación en salud; y en último lugar, se favoreció la construcción colectiva de las estrategias de comunicación, se brindaron herramientas para el diseño e implementación de campañas de comunicación, a través de una serie de preguntas que guiaron el proceso metodológico para operacionalizar los conceptos proporcionados y se propició un conversatorio donde cada uno de los miembros de la comunidad, debía reconocer las instancias y los aliados que se podían establecer para el desarrollo de la campaña de comunicación.

Se encuentran como limitantes la insuficiente experiencia en este tipo de enfoques, la limitación de tiempo y recursos para alcanzar objetivos de mayor plazo y la dificultad para medir el impacto final •

Agradecimientos. Las autoras expresan su agradecimiento en primer lugar a las instituciones financiadoras del "Modelos de movilización social con énfasis en la actividad física y estilos de vida saludables para reducir el sedentarismo en las regiones de Bogotá, Antioquia y Quindío”: Ministerio de Protección Social, Instituto 
Colombiano para el Desarrollo de la Ciencia y la Tecnología "Francisco José de Caldas-Colciencias", Universidad Nacional de Colombia y a la Fundación Ciudad Humana. En segundo lugar, al grupo de investigadores con los que participamos en el desarrollo del proyecto.

\section{REFERENCIAS}

1. Prieto A. Promoción de la salud y prevención de la enfermedad, desde la fisioterapia. Revisión conceptual. Revista Facultad Medicina Universidad Nacional de Colombia 2004; 52 (1):62-74.

2. La Iniciativa de comunicación. Bogotá: Gumucio-Dagron, A. Comunicación para la Salud: el Reto de la Participación. [Internet]. Disponible en: http://www. comminit.com/la/pensamientoestrategico/lasth/lasld-755.html.. Consultado: Junio 20 de 2004.

3. La Iniciativa de comunicación. Bogotá: Entrevista con. Armand Mattelart. (1 of 10). [Internet]. Disponible en: http://www.comminit.com/la/laint/sld4786.html. Consultado: Junio 16 de 2004.

4. Grupo de Investigación Cultura y Comunicación. Alsina, M. Elementos para una comunicación intercultural. Universitat Autónoma de Barcelona (UAB). [Internet]. Disponible en: http://www.aloj.us.es/ gicomcult/portada/37tx/5.htm. Consultado: Julio de 2004.

5. Pereira JM. Comunicación, promoción y salud. Signo y pensamiento 1994; 84-9.

6. Richard F. Population Services International-Informe Bianual 2001-2002. [Internet]. Disponible en: http:// www.psi.org/indexSP.html. Consultado: Junio 2004.

7. Wong F, Huhman M, Heitzler C, Asbury L, Bretthauer-Mueller R, McCarthy S, et al. VERB ${ }^{\mathrm{TM}}$ - a social marketing campaign to increase physical activity among youth. Preventing Chronic Disease. Disponible en: http://www.cdc.gov/pcd/issues/2004/jul/04_0043.htm. Consultado: Julio 2004.

8. Lloyd L. Comunicación y cambio conductual-el rol de COMBI. 8vo. Curso Internacional "El Dengue y la Fiebre Hemorrágica del Dengue. Todavía una Amenaza para la Salud Pública en las Américas”. Ciudad de La Habana, Cuba. 11-22 de Agosto del 2003.

9. Cohen L. Comunicación para PMTI. Introducción al modelo de comunicación para el desarrollo. Programme Communication, UNICEF New York. Presentado en OPS/OMS UNICEF Cuernavaca, México; 6-8 febrero, 2002.

10. Comunicación Macrointencional. [Internet]. Toro, JB; Rodríguez, MC. La comunicación y la movilización social en la construcción de bienes públicos". Disponible en http://www.comminit.com/la/lapm/sld-2035.html. Consultado: junio 30 de 2004.

11. Rizo LM. Iniciativa Centroamericana de Comunicación para el Desarrollo Sostenible, ICCADES - Centroamérica. 2001. En: Trees and People Newsletter No. $41 / 41$. 
12. La Iniciativa de comunicación. [Internet]. Bogotá: Moseley SF. Academia para el Desarrollo Educativo, AED-Global.

Disponible en: http://www.comminit.com/la/descripciones/lapdsglobal/ descripciones-1130.html. Consultado: julio 30 de 2004.

13. Irigoin M, Tarnapol P, Faulkner D, Coe G. Mapa de competencias de la comunicación para el desarrollo y el cambio social: Conocimientos, habilidades y actitudes en acción. Academy for Educational Development. Washington, 2002.

14. Miranda P. Usos y apropiaciones por parte de la comunidad de organizaciones emisoras miembros del sitio web Diario de la Sociedad Civil. Universidad Diego Portales. Santiago de Chile; 2002. 